\title{
MICROELEMENTS AND INHERITED METABOLIC DISEASES
}

\author{
Eliška Marklová \\ Charles University in Prague, Faculty of Medicine in Hradec Králové: Department of Paediatrics \\ Summary: In addition to the main groups of inherited metabolic diseases, including mitochondrial, peroxisomal and lyso- \\ somal defects, organic acidurias, porphyrias, defects of amino acids, saccharides and fatty acids metabolism, disorders of \\ transport and utilisation of microelements have also been recognized. Recent findings concerning hereditary hemo- \\ chromatosis (iron), Wilson and Menkes diseases (copper), molybdenum cofactor deficiency (molybdenum), defects of \\ cobalamine synthesis (cobalt) and acrodermatitis enteropathica (zinc) are reviewed.
}

Key words: Microelements; Inherited metabolic diseases

\section{Introduction}

Inherited metabolic disorders in children are not common; nevertheless their cumulative incidence is estimated to be 1 in every 500 newborns. Besides others, this group of diseases includes also the defects of transport and utilisation of minerals and microelements. The last act as catalysts for many biological reactions within the body, including muscle response, transmission of messages through the nervous system, production of hormones, digestion and the utilisation of nutrients in food.

Of the total 118 elements, 40-50 of them found in man, only 20 are known to be essential for the structure and function of the human body, the effect of the others being either uncertain or harmful (pollutants). The mineral nutrients are usually divided into macroelements, the most abundant substances dissolved in the extracellular and intracellular fluids, and microelements with daily requirement less than $100 \mathrm{mg}$ (21).

People can have deficient, adequate, or toxic intakes of any essential nutrient. Disturbances relevant to microelements might be caused by deficiency due to poorly balanced nutrition or by metabolic defects, both primary (inherited metabolic diseases) and secondary (digestive disorders and various chronic diseases).

Most common signs of the deficiency include growth retardation, nausea, diarrhoea, fatigue, muscle weakness, nervous and skin disorders, impaired immunity, difficulty in breathing, mouth and gum disorders (molybdenum), and smell and taste defects (zinc). The microelements supplied in higher concentration are toxic and the overdose symptoms are often similar to those following the element deficiency, e.g. nausea, vomiting, diarrhoea, dizziness, headaches, weight loss, shortness of breath. Considerable interactions in the process of the absorption have been described; e. g. high alcohol consumption leads to the higher urinary magnesium and zinc excretion and decreased zinc absorption, higher quantity of calcium in food reduces intestinal absorption of magnesium, iron, manganese and zinc, and antacids reduce iron and copper absorption (1).

Physiologically there is no need for extra purvey, but microelements supplementation is recommended under certain conditions, related to oxidative stress (smoking, exposure to chemicals, stress), digestive disorders, rapid growth (puberty), intensive exercise, ageing, pregnancy and lactation. Other indications might be special dietary regimes (diets rich in proteins, fats, carbohydrates, the vegan's or vegetarian's life style) and various secondary, but especially primary metabolic defects of microelements (22).

Several such disorders have been described in detail, namely hereditary hemochromatosis (iron), Wilson's and Menkes diseases (copper), molybdenum cofactor deficiency (molybdenum), defects of cobalamine synthesis (cobalt) and acrodermatitis enterica (zinc).

\section{Iron}

Only about $10 \%$ of iron (Fe) ingested is absorbed, animal sources being preferred. Acidic $\mathrm{pH}$ is necessary for $\mathrm{Fe}^{3+}$ reduction to $\mathrm{Fe}^{2+}$. Iron interferes with zinc and cobalt absorption.

The toxic effect of $\mathrm{Fe}$ overload is due to oxygen free radicals formation, leading to symptoms like diarrhoea, vomiting, cardiac failure and hepatotoxicity. Anaemia, brittle nails, constipation, impaired brain function, short breath, and tastes for odd foods (clay) are the main signs of iron deficiency. Humans have no physiological pathway for iron excretion, so that the intestinal absorption must be well regulated. 
Inherited metabolic defects are represented by a condition called hereditary hemochromatosis. High iron cumulation in many tissues (liver, pancreas, myocardium) due to its high intestinal absorption, exceeding the binding capacity for transferrin is very dangerous, because it leads to tissue damage.

The disease usually does not present itself until adulthood. The main signs include skin and other organ pigmentation, hemosiderosis, liver cirrhosis, hepatoma, carcinoma, diabetes, cardiac failure, arthritis and impotence, even if not all homozygotes manifest any symptoms. The cured patient has a normal life span, but the disease might be fatal if not diagnosed early or not treated aggressively.

The defect with an incidence 1:12 000 (more frequent in men) has an autosomal recessive trait. Two main (C282y and H63D) and other 15 rare mutations in the HFE (or HLA-H) gene on chromosome 6 expressed in duodenal crypts have been described since 1996. The other, recently described gene DMT1 (divalent metal transporter) and duodenal villi interact with the transferrin receptor. A distinct, non-HFE variant of the disease is juvenile hemochromatosis (impaired locus 1q). The other, mapped to $7 q 22$, contains the gene encoding transferrin receptor 2 (3).

Pathogenesis: Impaired iron absorption is a cascade of events: defected HFE protein, giving a false signal of a low iron concentration in cells influences the DMT1 protein, stimulating the iron uptake from a diet. Iron binds in excess to transferrin, reaches portal veins and finely leads to the liver and other organ cells death. Diabetes mellitus is the major endocrine disorder associated with the disease: Pancreatic $\beta$-cells damage by iron deposits and/or lack of chromium (a metal essential for insulin's proper function) due to its competition with iron absorption in gut is the speculated cause of this complication.

Diagnostics are based on the higher serum iron and ferritin levels, increased transferrin saturation (used as a screening test, pathological level being $>45 \%$ ), low iron-binding capacity, and especially DNA analysis.

Therapy based on the diet (uncooked sea-products, alcohol, minerals, and vitamin $\mathrm{C}$ restriction) or the chelating agents is usually not effective enough, so that phlebotomy or red cells apheresis must be used to prevent complications (450-500 $\mathrm{ml}$ of blood is removed every 1-2 weeks, until the ferritin level decreases to $10-20 \mathrm{mg} / \mathrm{l}$ ) (3).

\section{Copper}

About $30 \%$ of copper $(\mathrm{Cu})$ ingested is absorbed in the stomach and intestine. This metal makes a part of many $\mathrm{Cu}$-enzymes and plays an important role as an antioxidant, in iron transfer, and in haemoglobin synthesis. Copper deficiency may follow a higher intake of iron, molybdenum, zinc, milk or antacids in food and it manifests by symptoms such as weakness, diarrhoea and skin defects. Nausea, vomiting, dizziness, diarrhoea and headaches are the signs of copper overload.
There are two main inherited disorders of copper metabolism described so far, namely Menkes and Wilson's diseases.

Menkes disease results from impaired copper intestinal transport, caused by a deficit of extrahepatic Cu-ATPase.

Clinical symptoms of this neurodegenerative disorder are related to deficient levels of copper available, and include progressive mental deterioration, seizures, typical "facies" (cherubic, dolly face), peculiar (kinky, steely, brittle) hair, epilepsy, hypothermia, jaundice, bleeding, connective tissue abnormalities and skeletal changes. Death usually occurs in the first few years of life.

Pathogenesis: Copper deficit involves about $13 \mathrm{Cu}$-enzymes thus inducing diverse structural changes in hair, brain, bones, liver and arteries. Particularly deficient tyrosinase causes hair and skin depigmentations, and lysyl oxidase defect leads to splitting of arterial intima as a result of the defective elastin and collagen cross-linking. Monoamine oxidase and defective disulfide bonds in keratin are responsible for kinky hair; deficiency of cytochrome c oxidase leads to hypothermia, and decreased availability of ascorbate oxidase promotes skeletal deformities.

The incidence of this X-linked (males affected) defect runs about 1:300 000 (higher in Sardinia) with the most frequent mutations located on Xq12-q13.

Diagnostics are based on the low level of copper and ceruloplasmin in serum and high deoxypyridinoline in urine.

Therapy consisting in daily copper injections (physiological complex Cu-histidinate) is effective (more for the neurological than for the connective tissue abnormalities) only if started early, meaning between the ages of 3-13 years $(4,19)$.

Wilson's disease (hepatolenticular degeneration) is the other defect of transport of copper, the deficient enzyme being specified as the liver Cu-ATPase. The disease presents itself as a chronic liver disease (jaundice, abdominal swelling and pain, cirrhosis, vomiting of blood), KeyserFleischer ring in cornea, haemolysis and renal defects. Neurological symptoms include coordination and speech defects with possible bulbar paralysis and may be combined with psychiatric disorders. The liver defects manifest between 6-18 years of age, the neurological signs occur after the age of 20 years. The first symptoms are often misdiagnosed as infectious hepatitis or infectious mononucleosis.

Wilson's disease, affecting about 1 in 30000 people worldwide, is caused by gene ATP7B mutations on chromosome 13 (13q14.3) and transmitted as an autosomal recessive trait.

Pathogenesis: Deficiency of a copper-transporting ATPase protein leads to excessive cumulation of copper in the liver, basal ganglia and kidney, consequently to decreased excretion of copper in bile and low incorporation of copper into its transport-enzyme ceruloplasmin. Whether the primer is a low level of ceruloplasmin produced or it is the liver's inability to metabolise copper remains to be proved. 
Biochemical findings include a high level of copper in the urine and in liver biopsy, normal or decreased copper and ceruloplasmin in serum, along with a low copper incorporation into ceruloplasmin. The disease is always fatal if not diagnosed and treated early. The lifelong therapeutic approach consists in copper exclusion form food (mushrooms, nuts, chocolate, dried fruit, fish, liver), in supply of zinc-acetate, the copper absorption blocator, or an application of chelating agents such as penicillamine, trientine or possibly tetrathiomolybdate, and liver transplantation in the end $(5,19)$.

\section{Molybdenum}

Molybdenum (Mo) plays an important role as a part of Mo-pterine, cofactor of 3 enzymes: xanthine oxidase (XO, involves metabolism of purines), sulphite oxidase ( $\mathrm{SO}, \mathrm{me}-$ tabolism of cysteine) and aldehyde oxidase (AO, conversion of aldehydes to acids). The main role of the enzymes lies in detoxification and synthesis of intermediates.

A deficiency, usually due to the parenteral diet low in Mo, manifests as visual problems, tachycardia, headache, mental disturbances, and mouth and gum disorders. Symptoms of toxicity include diarrhoea, growth retardation, gout and copper deficit.

Inherited metabolic defects leading to individual enzyme deficiency might be isolated or combined: deficiencies of $\mathrm{XO}$, SO and molybdenum cofactor are described. Although XO deficiency might be relatively benign, patients with isolated deficiencies of SO or molybdenum cofactor exhibit similarly severe abnormalities probably caused by the toxicity of sulphite and/or a deficit of inorganic sulphate available, especially in the brain. A defect of the third enzyme, AO is not combined with special symptoms.

Xanthinuria. Xanthine oxidase, catalysing the uric acid production from xanthine, is a complex of flavines, molybdenum, iron and sulphite cofactor.

Clinical symptoms of the enzyme deficit manifesting itself in early childhood include hematuria, nephrolithiasis (X-ray contrast), renal failure, arthropathy and myopathy, but as much as $50 \%$ of homozygotes for the defect might be fully asymptomatic.

The disease, with a frequency 1 in 40000 newborns, shows an autosomal recessive type of inheritance implying mutation on chromosome 2 .

Diagnostics are based on low uric acid and high xanthine and hypoxanthine concentrations in the blood and urine.

Symptomatic therapy consists of low-purines diet, drinking regime, and lithotripsy (7).

Isolated deficit of sulphite oxidase leads to impaired metabolism of sulphur-containing amino acids (cysteine, methionine, homocysteine, taurine) in the liver, brain and kidney.

Clinical symptoms include lens subluxation accompanying seizures and diffuse neurological disease in infancy.
The cause of this rare genetic condition is a point mutation, causing very low enzyme activity due to changes of Mo-centrum geometry.

Diagnostics are based on a positive sulphite test (in fresh urine) and plasma amino acids, namely increased taurine and sulphocysteine. Mutation analysis can allow accurate prenatal screening (2).

Molybdenum-cofactor deficiency is a fatal disorder clinically manifesting shortly after birth with profound neurological and psychiatric problems, mental retardation, microscopic haematuria and ocular lens dislocation with Marfan-like habitus. This condition might be an easily missed cause of intractable seizures in the neonatal period.

The defect is an autosomal recessive disorder with genetic heterogeneity. A linkage of a defective gene to an 8 -cM region on chromosome 6 p21.3 was demonstrated.

The biochemical abnormalities include very low levels of uric acid in serum and urine (low XO activity) and low inorganic sulphate levels in urine (low SO activity). Elevated S-sulphocysteine and taurine (in plasma and urine), high excretion of xanthine, hypoxanthine and the oxidized derivative of a molybdopterin precursor (precursor Z) in urine are typical findings. A defect of terminal enzyme for converting precursor $\mathrm{Z}$ to active molybdopterin is supposed.

Molybdenum cofactor deficiency is probably frequently underdiagnosed due to the lack of specific clinical features. Screening of infants at risk for the presence of urinary sulphites and/or serum hypouricemia is rapid and inexpensive.

No specific therapy has been recommended so far. Prenatal diagnostics is available $(17,18)$.

\section{Cobalt}

Cobalt $(\mathrm{Co})$ is not absorbed in natural form, but makes a base of cobalamin ( $\mathrm{Cbl}$, vitamine $\left.\mathrm{B}_{12}\right)$, which plays a role as a coenzyme of two important enzymes: methionin-synthase (cytosolic methyl-Cbl) and methylmalonyl-CoA-mutase (mitochondrial adenosyl-Cbl formation).

A deficiency of $\mathrm{Cbl}$ might occur under poor nutrition, especially in children of breast-feeding mothers on a $B_{12}$-deficient vegan's diet. Cbl toxicity appears as diarrhoea, fatigue and polycythemia.

Cobalamins are essential biological compounds structurally related to haemoglobin and the cytochromes. Although only microorganisms synthesize the basic cobalamin molecule, all mammalian cells can convert this into the coenzymes adenosylcobalamin (AdoCbl) and methylcobalamin ( $\mathrm{MeCbl})$.

Several genetic defects have been described, making two main groups: 1) disorders of $\mathrm{Cbl}$ absorption and transport, and 2) disorders of intracellular Cbl metabolism. These conditions are mostly inherited as an autosomal recessive trait with various mutations, total incidence is approximated as $0.8 / 100000$. 
Pathogenesis is similar in all types of defects: deficiency of $\mathrm{MeCbl}$ and AdoCbl precursors leads to deficiency of the coenzymes causing defective enzyme reaction.

Ad 1) Disorders of absorption and transport of cobalamin may be caused by: a) gastric malabsorption (defect of $\mathrm{Cbl}$ release from proteins), b) deficiency of R-glycoprotein (which binds $\mathrm{Cbl}$ released in the stomach), c) deficiency of intrinsic factor (IF) or its receptor (cubilin), d) deficiency of transcobalamin II, or e) gut malabsorption (ImerslundGräsbeck syndrome).

Clinical symptoms include failure to thrive, lethargy, hypotonia, vomiting, developmental delay, megaloblastic anemia and progressive neuro(myelo)encephalopathy. Defects appear in toddlers or pre-school children, the defect of IF much sooner.

Diagnostics are based on a decrease of $\mathrm{Cbl}$ and higher levels of both methylmalonic acid and homocysteine in urine. Low transcobalamine II is typical for the type (d).

Therapy consists of daily application of hydroxy-Cbl (or cyano-Cbl) in a dose of $1 \mathrm{mg} /$ day i.m., or the IF application in the type (c).

Ad 2) Disorders of intracellular metabolism of cobalamin. There exist several forms of $\mathrm{Cbl}$ intermediates giving names to individual subgroups of defects: a) Cbl-F defect - disorder of lysosomal Cbl release, b) Cbl-C and Cbl-D defects - cytosolic disorders of AdoCbl a MeCbl synthesis, c) Cbl-E and Cbl-G defects - disorder of MeCbl synthesis, and d) Cbl-A and Cbl-B defects - disorder of mitochondrial AdoCbl synthesis.

Clinical signs of the type (a) and (b) resemble the transport defects (low haemoglobin, trombocytopenia, anaemia, high homocystein and methylmalonic acid). Failure to thrive, growth retardation, hypo-/hypertonia, epilepsy, progressive encephalo/neuropathy and megaloblastic anemia are characteristic for type (c). The type (d) leads to methylmalonic aciduria, resembling the other type, caused by the methylmalonic acid-mutase deficiency (vomiting, coma, and death following by acidosis, ketosis, hyperammonaemia, hyperglycinemia, organic aciduria and hypoglycaemia. All defects manifest during the first months of life, sometimes even occuring neonatally.

High methylmalonic acid in urine is characteristic of types (a), (b) and (d), increased plasma homocysteine is typical for types (a), (b) and (c). Both Cbl and transcobalamine II are normal.

Application of $1 \mathrm{mg} /$ day i. m. of hydroxy-Cbl (cyano$\mathrm{Cbl}$ is less practical), betain and folate presents an effective therapy for those diseases.

\section{Zinc}

This metal, present in all tissues, plays an important role in cell growth and differentiation, immunity, taste and alcohol breakdown. It is an important component of insulin and a cofactor of multiple enzymes in metabolism of neurotransmitters and various hormones. Zinc ( $\mathrm{Zn})$ absorp- tion in the upper intestine may be influenced by the presence of fibres (binding) and calcium (competition) in food.

Zinc deficiency, prevalent in population on cereal proteins, malabsorption conditions, liver cirrhosis and renal diseases, manifests itself as fatigue, brittle nails, infections, dwarfism and impaired growth, healing and fertility. An overload presents as nausea, vomiting and renal failure.

The only inherited metabolic defect connected with zinc recognised so far is called acrodermatitis entheropathica.

Clinical signs include skin rash, mucous lesions, total alopecia, diarrhoea, mood changes and irritability (but all of them are present in only $20 \%$ of the cases). Their intermittent occurrence and the vast range of other, also misleading symptoms, may cause a diagnostic problem.

Mutation of this autosomal recessive disorder was localised on chromosomal region $8 \mathrm{q} 24.3$.

Pathogenesis is still not clear: Defect of zinc transport in gut and other tissues leads to a defect of $\mathrm{Zn}$ utilisation resulting in decreased synthesis of prostaglandines. Other results suggest an intracellular defect in $\mathrm{Zn}$ metabolism rather then impairment of the absorption. The hypothesis that the exocrine pancreas modulates $\mathrm{Zn}$ absorption in the jejunum might help in explaning of the conflicting dates of this inherited defect. Transient acrodermatitis enteropathica due to a decreased $\mathrm{Zn}$ level in breast milk has been described in two children. Also bovine hereditary Zn deficiency with an absorption defect (Adema disease) has been recognized.

Low serum zinc and alkaline phosphatase activity are used as the diagnostic criterion. Zinc substitution ( $\mathrm{Zn}$-sulphate: $10 \mathrm{mg} / \mathrm{kg} /$ day) is effective in the therapy $(14,15,16$, 20).

\section{Conclusions}

Several other genetic defects derived from impaired utilization of trace minerals (selenium and manganese, e.g.) have been considered, but the pathogenesis is still not clear. As the number of known inherited metabolic diseases gradually increases, new defects of microelements will probably be recognised in the future. However, since the clinical picture is usually non-specific and the detection of their deficiency or accumulation requires application of special analytical techniques, the diagnostics might be rather difficult.

\section{Acknowledgments}

Contributed as a lecture to the CEEPUS Summer University - Gastrointestinal tract and liver; multidisciplinary approach, July 20 - 29, 2001, Zadar, Croatia.

\section{References}

1. Driskell JA. Vitamin and Mineral Toxicities in Adults. In: Foods and Nutrition NebFact, Nebraska-Lincoln 1992:128-33.

2. Edwards MC, Johnson JL, Marriage B et al. Isolated sulfite oxidase deficiency: review of two cases in one family. Ophthalmology 1999;106:1957-61. 
3. Galy B. Hereditary haemochromatosis: more causative genes [Journal Club] TIBS 2001;26:473.

4. George DH, Casey RE. Menkes disease after copper histidine replacement therapy: case report. Pediatr Dev Pathol 2001;4:281-8.

5. Gow PJ, Smallwood RA, Angus PW, Smith AL, Wall AJ, Sewell RB. Diagnosi of Wilson's disease: an experience over three decades. Gut 2000;46:415-9.

6. Hanson EH, Imperatore G, Burke W. HFE gene and hereditary hemochromatosis: a HuGE review. Human Genome Epidemiology. Am J Epidemiol 2001; 154:193-206.

7. Ichida K, Amaya Y, Kamatani N, Nishino T, Hosoya T, Sakai OJ. Identification of two mutations in human xanthine dehydrogenase gene responsible for classical type I xanthinuria, Clin Invest 1997;99:2391-7.

8. Julian F. B. Mercer JFB. The molecular basis of copper-transport diseases. Trend in Molecular Medicine 2001;7:64-69.

9. Koolman J, Röhm KH. Color atlas of biochemistry. Stuttgart, New York: Thieme, 1996:435.

10. Krasowska D. Acrodermatitis enteropathica-congenital zinc deficiency syndrome. Wiad Lek 1992;45:454-7.

11. Krumbach CJ, Ellis DR, Driskell JA. A report of vitamin and mineral supplement use among university athletes in a division I institution. Int J Sport Nutr 1999;9:416-25.

12. Linnell JC, Bhatt HR. Inherited errors of cobalamin metabolism and their management, Baillieres Clin Haematol 1995;8:567-601.

13. Lombeck I, Bremer HJ. Primary and secondary disturbances in trace element metabolism connected with genetic metabolic disorders. Nutr Metab 1977;21 49-64

14. Machen M, Montgomery T, Holland R et al. Bovine hereditary zinc deficiency: lethal trait A 46. J Vet Diagn Invest 1996:8:219-27.

15. Niemi KM, Anttila PH, Kanerva L, Johansson E. Histopathological study of transient acrodermatitis enteropathica due to decreased zinc in breast milk. J Cutan Pathol 1989;16:382-7.
16. Sandström B, Cederblad A, Lindblad BS, Lönnerdal B. Acrodermatitis enteropathica, zinc metabolism, copper status, and immune function. Arch Pediat Adolesc Med 1994;148:980-5.

17. Shalata A, Mandel H, Reiss J, et al. Localization of a gene for molybdenum cofactor deficiency, on the short arm of chromosome 6, by homozygosity mapping. Am J Hum Genet 1998;63:148-54.

18. Slot HM, Overweg-Plandsoen WC, Bakker HD et al. Molybdenum-cofactor deficiency: an easily missed cause of neonatal convulsions. Neuropediatrics 1993;24:139-42.

19. Strausak D, Mercer JFB, Dieter HH, Stremmel W and Gerd Multhaup G. Copper in disorders with neurological symptoms: Alzheimer's, Menkes, and Wilson diseases. Brain Research Bulletin 2001;55:175-85.

20. Van Wouwe JP, Uijlenbroek JJ. The role of the pancreas in the regulation of zinc status. Biol Trace Elem Res 1994;42:143-9.

21. Zimmermann M. Micronutrients in health and disease. Stuttgart, New York: Thieme, 2001:240.

22. Zschocke J, Hoffmann GF. Vademecum metabolicum, Milupa GmbH \& Co KG 1999:111.

Submitted April 2002.

Accepted September 2002.

MUDr. Eliška Marklová, CSc., Teaching Hospital in Hradec Králové, Department of Paediatrics, Sokolská 408, 50005 Hradec Králové, Czech Republic. e-mail: marklova@lfhk.cuni.cz 